\title{
The modification of a node-to-node algorithm for the modelling of beam connections in RFEM and SCIA using the explicit method
}

\author{
Hynek Štekbauer ${ }^{1, *}$ and Zbyněk $\mathrm{Vlk}^{1}$ \\ ${ }^{1}$ Brno University of Technology, Faculty of Civil Engineering, Institute of Structural Mechanics, \\ Veveř́ 331/95, 60200 Brno, Czech Republic
}

\begin{abstract}
The "node-to-node" (NTN) algorithm is one of the simplest and oldest contact discretization methods [1]. It is, therefore, used in cases where a more sophisticated method is not needed. Beam connections between 1D elements are a great example of an area of application of NTN discretization. Each connection is modelled by a hinge at the end of every connected 1D element. The explicit method is important with regard to many linear and non-linear dynamics problems. The central difference method is usually used in the Finite Element Method to solve explicit schemes. This and similar methods suffer significant errors if the time step exceeds the critical time step, which is directly derived from the element stiffness and tends towards zero as connected nodes get closer to each other. This is very inconvenient for NTN contacts, as with them there is usually only a small distance (or even zero distance) between the nodes and a rigid connection in a particular direction. This paper presents a modification that enables hinges that are rigid in a particular direction to be calculated using the explicit method.
\end{abstract}

\section{Introduction}

\subsection{The central difference method}

The basic equation of motion for dynamics in the central difference method at time $t_{n}$ is

$$
\mathbf{M} \ddot{\mathbf{u}}_{n}=\mathbf{P}_{n}-\mathbf{F}_{n}-\mathbf{C} \dot{\mathbf{u}}_{n},
$$

where $\mathbf{M}$ is the diagonal mass matrix, $\mathbf{P}_{n}$ is the vector of external forces, $\mathbf{F}_{n}$ is the vector of internal forces, and $\mathbf{C}_{n}$ is the diagonal damping matrix [2]. Vectors $\mathbf{P}_{n}$ and $\mathbf{F}_{n}$ are usually represented by $\mathbf{R}_{n}$

$$
\mathbf{R}_{n}=\mathbf{P}_{n}-\mathbf{F}_{n} .
$$

Acceleration $\ddot{\mathbf{u}}_{n}$ can be expressed as

$$
\ddot{\mathbf{u}}=\mathbf{M}^{-1}\left(\mathbf{R}_{n}-\mathbf{C u}_{n-1}\right) .
$$

\footnotetext{
*Corresponding author: stekbauer@seznam.cz
} 
Due to the diagonal matrices $\mathbf{M}$ and $\mathbf{C}_{n}$, the system of equations can be decomposed into the solution of $N$ independent equations for every degree of freedom in every node

$$
\begin{gathered}
\ddot{u}_{n}{ }^{j}=\left(R_{n}{ }^{j}-C_{n}{ }^{j} \cdot \dot{u}_{n-1}\right) / m^{j} \\
\Delta \dot{u}^{j}=\ddot{u}_{n}{ }^{j} \cdot \Delta t_{n}, \\
\dot{u}_{n+1 / 2}^{j}=\dot{u}_{n-1 / 2}^{j}+\Delta \dot{u}^{j}, \\
u_{n+1}^{j}=u_{n}^{j}+\dot{u}_{n+1 / 2}^{j} \cdot \Delta t_{n+1 / 2},
\end{gathered}
$$

where

$$
\Delta t_{n+1 / 2}=\frac{\Delta t_{n}+\Delta t_{n+1}}{2}
$$

and $j$ takes values from 1 to $N$. This approach is fast and easy to parallelize. Rigidly supported nodes have prescribed deformations. The critical time step can be calculated from wave propagation velocity in beam $c$ using the modulus of elasticity $E$ and the density $\rho$

$$
c=\sqrt{\frac{E}{\rho}} .
$$

The Courant-Friedrichs-Levy stability criterion for the time needed for the wave to propagate through a beam of length $l$

$$
\Delta \mathrm{t} \leq \frac{l}{c}
$$

\subsection{NTN model of a hinge}

NTN discretization is a simple and stable discretization method for conforming meshes [3] and is used in RFEM and SCIA for hinge modelling. An NTN contact consists of two nodes (slave and master), and a transformation matrix that characterizes hinge direction (Fig. 1).

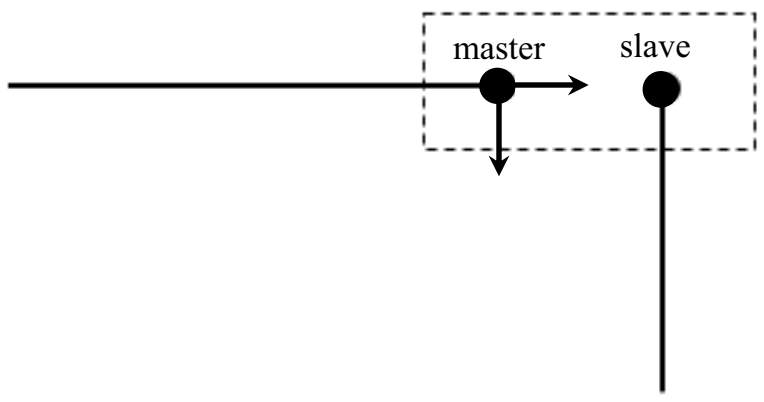

Fig. 1. The NTN contact (slave and master) and a transformation matrix that characterizes hinge direction.

The stiffness matrix of a hinge using the penalty stiffness method is given by 


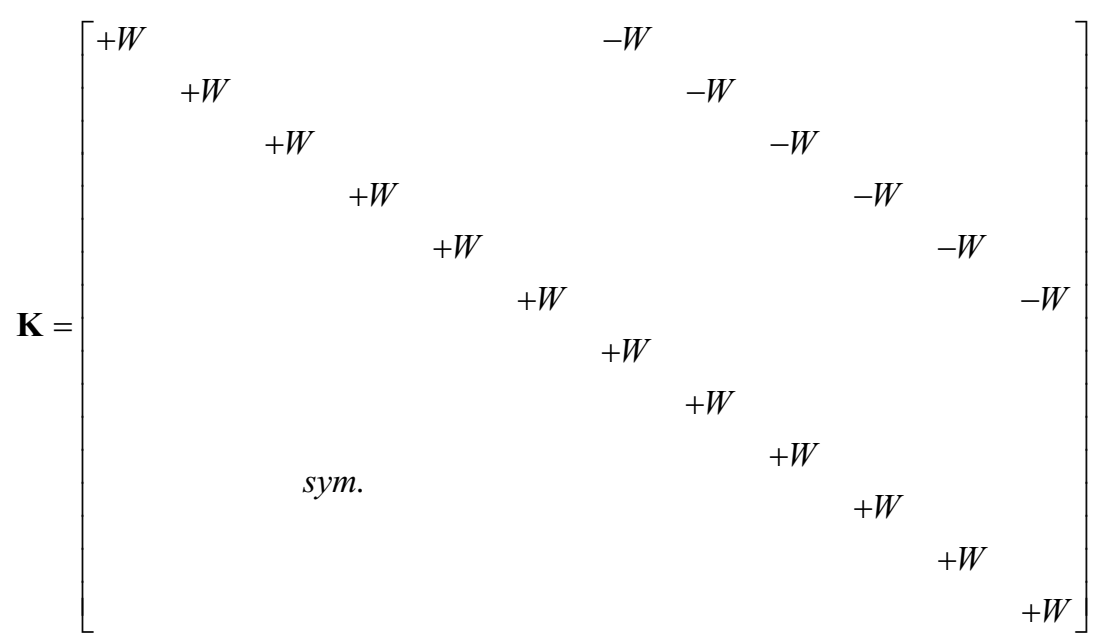

where $W$ is the penalty weight for a rigid directions of a hinge. The penalty weight $W$ can be replaced in a required degree of freedom

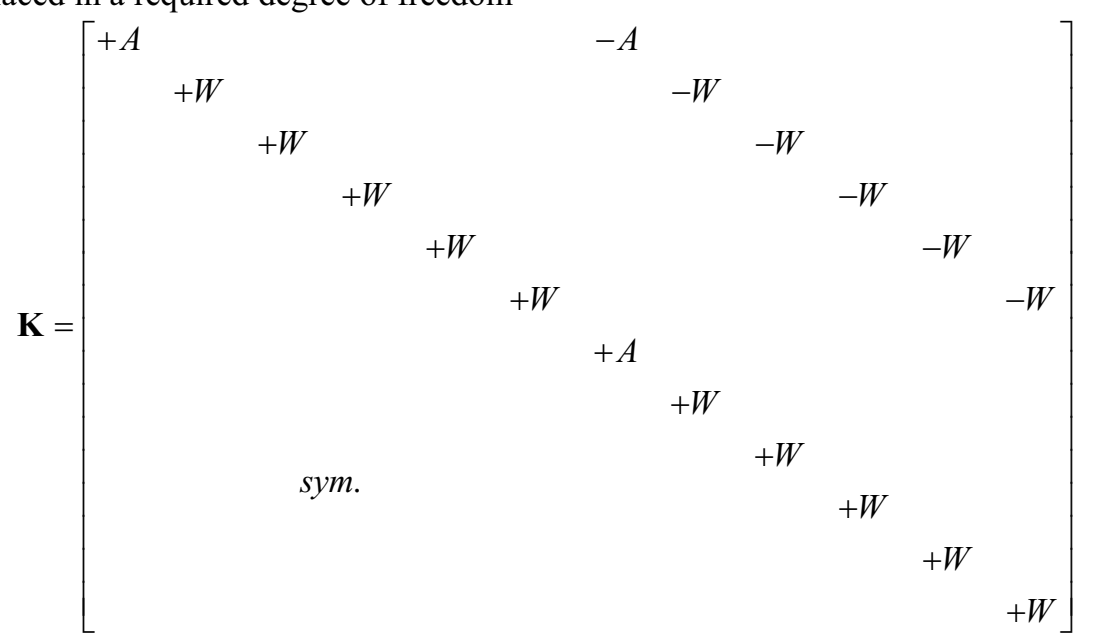

where $A$ is a stiffness characteristic for direction $u_{x}$. The transformation matrix of a hinge

$$
\mathbf{T}_{R}=\left[\begin{array}{ll}
\mathbf{T} & \mathbf{0} \\
\mathbf{0} & \mathbf{T}
\end{array}\right]
$$

is composed of direction rotation matrices $\mathbf{T}$ between the global and local coordinate system

$$
\mathbf{T}=\left[\begin{array}{lll}
C_{x, X} & C_{x, Y} & C_{x, Z} \\
C_{y, X} & C_{y, Y} & C_{y, Z} \\
C_{z, X} & C_{z, Y} & C_{z, Z}
\end{array}\right]
$$

where $C_{i, J}$ means the cosine between axes $\mathrm{i}$ and $\mathrm{J}$. The distance between nodes is taken into account in the transformation matrix extended by the eccentricity matrix $\mathbf{E}$

$$
\mathbf{T}_{R E}=\left[\begin{array}{cc}
\mathbf{T} & \mathbf{T E} \\
\mathbf{0} & \mathbf{T}
\end{array}\right] .
$$


Eccentricity matrix

$$
\mathbf{E}=\left[\begin{array}{ccc}
0 & e_{z} & -e_{y} \\
-e_{z} & 0 & e_{x} \\
e_{y} & -e_{x} & 0
\end{array}\right]
$$

consists of eccentricities and is skew symmetric.

Generally, there are three types of hinges that are used in structures. First, there is a rigid hinge that simulates the infinitely stiff connection of two nodes. A rigid hinge alone has no meaning in the Finite Element Method because this type of connection can easily be modelled by elements with a common node, but it is needed if only some selected degrees of freedom are not rigid. The second type is a linear hinge with given stiffness in a particular direction. The third and most general type is the nonlinear hinge, whose behaviour can be described by a function or by the dependence of one direction on another direction (i.e. friction).

\section{The modification of the NTN algorithm}

In this section, the modification needed for the presented NTN hinge model to work properly in the explicit method is described. Upper script $j$ is used to label the first and second node of the contact and takes the values 1 and 2 .

$$
\begin{gathered}
\mathbf{R}_{G}^{j}=\left(\begin{array}{c}
r_{u x} \\
r_{u y} \\
r_{u z} \\
r_{\varphi x} \\
r_{\varphi y} \\
r_{\varphi z}
\end{array}\right) \\
\mathbf{R}_{L}^{j}=\mathbf{T}_{R} \cdot \mathbf{R}_{G}^{j} \\
\mathbf{R}_{D U M}^{j}=\mathbf{R}_{L}^{j}
\end{gathered}
$$

For all rigid directions $r d$ of $\mathbf{R}_{L}^{j}$

$$
\begin{aligned}
& \mathbf{R}_{L}^{1}(r d)=\mathbf{R}_{L}^{1}(r d)+\mathbf{R}_{D U M}^{2}(r d) \\
& \mathbf{R}_{L}^{2}(r d)=\mathbf{R}_{L}^{2}(r d)+\mathbf{R}_{D U M}^{1}(r d)
\end{aligned}
$$

where $r d$ can take values between 1 (for $r_{u x}$ ) and 6 (for $r_{\varphi z}$ ) inclusive.

$$
\mathbf{R}_{G}^{j}=\mathbf{T}_{R}^{T} \cdot \mathbf{R}_{L}^{j}
$$

Similar operations are performed for masses

$$
\begin{aligned}
& \mathbf{M}_{L}^{j}=\left[\begin{array}{cccccc}
m_{L x} & & & & & \\
& m_{L y} & & & & \\
& & m_{L z} & & & \\
& & & m_{L \varphi x} & & \\
& & & & m_{L \varphi y} & \\
& & & & & m_{L \varphi z}
\end{array}\right] \\
& \mathbf{M}_{L}^{j}=\mathbf{T}_{R} \cdot \mathbf{M}_{G}^{j} \cdot \mathbf{T}_{R}{ }^{T} .
\end{aligned}
$$

Matrix $\mathbf{M}_{L}^{j}$ is not unconditionally diagonal, which is why it needs to be diagonalized if needed. 
For all rigid directions $r d$ of $\mathbf{M}_{L}^{j}$

$$
\mathbf{M}_{D U M}^{j}=\mathbf{M}_{L}^{j}
$$

$$
\begin{aligned}
& \mathbf{M}_{L}^{1}[r d, r d]=\mathbf{M}_{L}^{1}[r d, r d]+\mathbf{M}_{D U M}^{2}[r d, r d] \\
& \mathbf{M}_{L}^{2}[r d, r d]=\mathbf{M}_{L}^{2}[r d, r d]+\mathbf{M}_{D U M}^{1}[r d, r d]
\end{aligned}
$$

where $r d$ can take values between 1 (for $m_{u x}$ ) and 6 (for $m_{\varphi z}$ ) inclusive. The masses are then transformed back to the global coordinate system and diagonalized if needed

$$
\mathbf{M}_{G}^{j}=\mathbf{T}_{R}{ }^{T} \cdot \mathbf{M}_{L}^{j} \cdot \mathbf{T}_{R} .
$$

After such a modification, deformations are calculated using the central difference method (Eq. 4-8). Up until this point, only some of the necessary modifications have been presented. These are modifications which are good for hinges that are linear or rigid and without rigid support in any direction. A special treatment needs to be added for supported hinges and nonlinear hinges that should act in a rigid manner under certain circumstances. Deformations calculated by the central difference method are transformed to the local coordinate system of the hinge and examined for these two special cases.

$$
\begin{gathered}
\mathbf{U}_{G}^{j}=\left(\begin{array}{l}
u_{x}{ }^{j} \\
u_{y}{ }^{j} \\
u_{z}{ }^{j} \\
\varphi_{x}{ }^{j} \\
\varphi_{y}{ }^{j} \\
\varphi_{z}{ }^{j}
\end{array}\right) \\
\mathbf{U}_{L}^{j}=\mathbf{T}_{R} \cdot \mathbf{U}_{G}^{j} \\
\mathbf{U}_{D U M}^{j}=\mathbf{U}_{L}^{j}
\end{gathered}
$$

At first, the identification of supported hinge nodes in a particular direction is performed. Then, deformations from the rigidly supported node can be assigned to the other node in every rigid direction $r d$ of a hinge. If the second node is rigidly supported

$$
\mathbf{U}_{L}^{1}(r d)=\mathbf{U}_{D U M}^{2}(r d),
$$

and vice versa if the first node is rigidly supported

$$
\mathbf{U}_{L}^{2}(r d)=\mathbf{U}_{D U M}^{1}(r d) \text {. }
$$

After that, nonlinear hinges described by a function are evaluated. The user can define the limit stiffness which is taken as rigid.

A rigid branch of a function that is stiffer then the limit stiffness can be seen in Fig. 2. If any displacement $d$ between contact nodes exceeds the limit value $d_{\lim }\left(d_{\lim }=1 \mathrm{~mm}\right.$ for Fig. 2), where $d$ is the $x$-th element of a vector $\mathbf{D}$

then local displacements are recalculated

$$
\mathbf{D}=\mathbf{U}_{L}^{2}-\mathbf{U}_{L}^{1},
$$

$$
\begin{aligned}
& \mathbf{U}_{L}^{1}(x)=\mathbf{U}_{L}^{1}(x) \cdot \frac{d_{\lim }}{d}, \\
& \mathbf{U}_{L}^{2}(x)=\mathbf{U}_{L}^{2}(x) \cdot \frac{d_{\lim }}{d},
\end{aligned}
$$

and transformed back to the global coordinate system

$$
\mathbf{U}_{G}^{j}=\mathbf{T}_{R}^{T} \cdot \mathbf{U}_{L}^{j}
$$




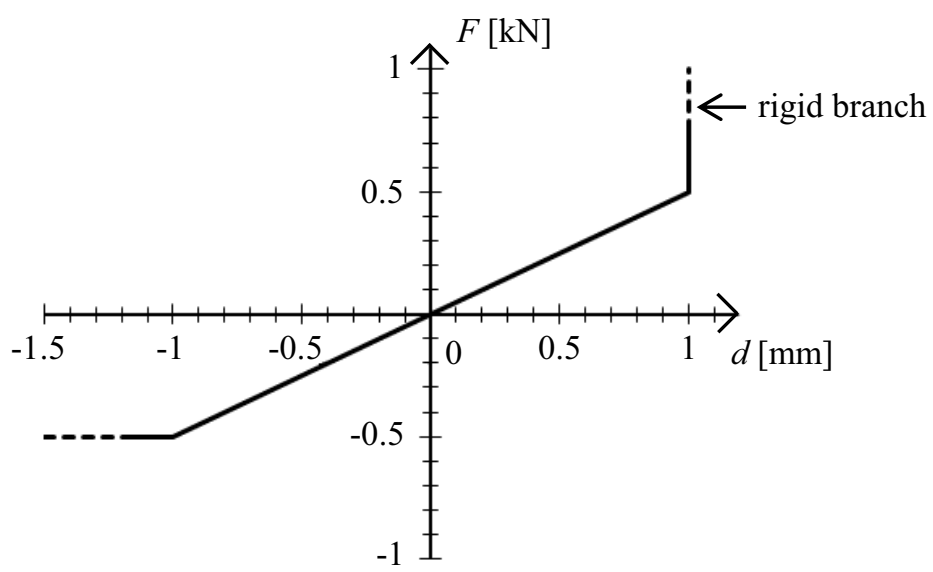

Fig. 2. Nonlinear hinge function with a rigid branch (a branch that is stiffer than the limit stiffness) showing the dependency of force $F$ on a certain displacement $u$. The similar dependency of moment on rotation can be prescribed.

Accelerations and velocities need to be recalculated for modified displacements $\mathbf{U}_{G}^{j}$ using Eq. 5-7 backwards.

$$
\begin{gathered}
\dot{u}_{n+1 / 2}^{j}=\frac{u_{n+1}^{j}-u_{n}^{j}}{\Delta t_{n+1 / 2}} \\
\Delta \dot{u}^{j}=\dot{u}_{n+1 / 2}^{j}-\dot{u}_{n-1 / 2}^{j} \\
\ddot{u}_{n}{ }^{j}=\frac{\Delta \dot{u}^{j}}{\Delta t_{n}}
\end{gathered}
$$

\section{Conclusions}

The modification of an NTN algorithm for the modelling of beam connections in the explicit method is proposed. The modification is divided into two sections, the first of which involves the recalculation of nodal forces and masses based on the rigid local directions of a contact before the solution of equations. The second part is needed for the supported contacts and nonlinear contacts that act in a rigid manner under certain circumstances. Deformations calculated by the central difference method are transformed to the local coordinate system of the contact and modified to fulfil contact conditions and prevent solution instability at the same time.

This research was partially supported by the project FAST-J-17-4720.

\section{References}

1. G. Zavarise, L. De Lorenzis, Comput. Method Appl. M. 198, 3428 (2009)

2. K. J. Bathe, Finite Element Procedures (Prentice Hall, New Jersey, 2009)

3. V. A. Yastrebov, Numerical Methods in Contact Mechanics (ISTE/Wiley, Oxford, 2013) 